\title{
Ferramentas de computação visual para apoio ao treinamento de expressões faciais por autistas: uma revisão de literatura
}

\author{
Thiago Pradi $^{1}$, Luciano Silva ${ }^{1}$, Olga R. P. Bellon ${ }^{1}$, Gustavo M. S. Dória ${ }^{2}$ \\ ${ }^{1}$ Departamento de Informática - Grupo IMAGO de Pesquisa \\ Universidade Federal do Paraná - Curitiba, PR - Brasil. \\ ${ }^{2}$ Departamento de Medicina Forense e Psiquiatria \\ Universidade Federal do Paraná - Curitiba, PR - Brasil \\ tpradi@inf.ufpr.br, \{luciano, olga\}@ufpr.br, gustavodoria@brturbo.com.br
}

\begin{abstract}
Advances on research and application of visual computing on health sciences have become important allies in solving problems faced by individuals with Autism Spectrum Disorder (ASD). The difficulty in recognizing and producing emotions through the face often makes the social relationships of this population less adaptive and functional, which prejudice their autonomy. This paper reviews and discusses state-of-the-art works that use visual computing resources to provide solutions to challenges related to ASD. From this broad study, we raise questions and provide future directions for development of new tools to train recognition and production of facial expressions by children with autism, aiming both diagnosis and treatment.
\end{abstract}

Resumo. Os avanços nas pesquisas e aplicações de computação visual em ciências da saúde se tornaram importantes aliados na resolução de problemas enfrentados por indivíduos com Transtorno do Espectro Autista (TEA). Esta população apresenta limitações em reconhecer e expressar emoções através da face, o que torna suas relações sociais menos adaptativas e funcionais. Este artigo apresenta uma análise de trabalhos que utilizam recursos de computação visual para apoiar o incremento da capacidade de reconhecimento de emoções expressas pela face. A partir deste estudo, levanta-se questões e indica-se direções futuras para o desenvolvimento de novas ferramentas para treinar $o$ reconhecimento e a produção de expressões faciais em crianças com autismo.

\section{Introdução}

O autismo pertence ao grupo dos transtornos do neurodesenvolvimento, nos quais ocorre uma ruptura em processos fundamentais de socialização, comunicação e aprendizado do indivíduo [Klin 2006]. Novos estudos [Matson and Kozlowski 2011] apontam que o número de casos diagnosticados de autismo vem crescendo nos últimos anos. Em pesquisa recente do Centers for Disease Control and Prevention (CDC), estimou-se uma prevalência de uma em cada quarenta e cinco crianças, nos EUA, para o transtorno [Zablotsky et al. 2015]. Este crescimento está relacionado a diversos fatores, sobretudo às mudanças nos critérios de diagnóstico e conscientização da população em geral [Matson and Kozlowski 2011].

Entre as limitações apresentadas por indivíduos com TEA, pode-se citar a dificuldade na tarefa de reconhecimento e produção de expressões faciais 
[Baron-Cohen et al. 1985]. Paul Ekman [Ekman and Friesen 1978] sugere que as habilidades para reconhecer e expressar emoções através da face são intrínsecas na raça humana. As emoções expressas através da face apresentam um papel importante na comunicação e interação entre seres humanos. Desde os primeiros meses de vida, recémnascidos já interagem a partir de expressões faciais, utilizando-as como dicas essenciais para entendimento da comunicação [Picard et al. 2001].

Apesar do autismo não ter cura, abordagens terapêuticas podem ser eficazes na melhora do quadro, conferindo maior qualidade de vida e prevenindo o agravamento da doença. Por exemplo, recursos tecnológicos vêm sendo explorados com sucesso no caso de autistas que apresentam facilidade no uso de computadores, tablets e similares. Dentre essas iniciativas, um dos focos é incrementar a assertividade no reconhecimento e produção de expressões faciais.

Este trabalho apresenta uma revisão de trabalhos que utilizam recursos de computação visual para o treinamento em reconhecimento e produção de expressões faciais por autistas. As publicações inclusas nesta revisão foram avaliadas sob um conjunto de cinco critérios definidos pelos autores, dando ênfase nas contribuições dos recursos e métodos utilizados para o incremento desta habilidade. Este trabalho está organizado como se segue. A Seção 2 apresentando um comparativo das principais iniciativas que utilizam recursos computacionais para treinamento no reconhecimento e produção de expressões faciais. A Seção 3 discute os achados levantados com o presente estudo comparativo. A Seção 4 conclui o texto e sugere direções futuras.

\section{Treinamento em reconhecimento e produção de expressões faciais por autistas}

Sabe-se que autistas apresentam limitações que prejudicam a formulação da sua própria Teoria de Mente [Baron-Cohen et al. 1985]. A Teoria de Mente é a capacidade do indivíduo em atribuir estados mentais a si próprios e aos outros, diferenciar estes estados entre si, e interpretá-los. Conforme experimento [Gordon et al. 2014], a limitação que autistas apresentam para produzir expressões faciais não tem ligação com problemas motores ou musculares do rosto, mas sim com uma desconexão entre o estado mental de uma emoção e sua representação facial. Em [Tanaka et al. 2012] argumenta-se que autistas apresentam dificuldades em generalizar expressões faciais entre indivíduos, o que acaba agravando esse déficit.

Diversas pesquisas [Gordon et al. 2014, Tanaka et al. 2010, Deriso et al. 2012, Bölte et al. 2002, Silver and Oakes 2001] comprovam através de experimentos que é possível treinar as habilidades de reconhecimento e produção de expressões faciais por autistas, com a ajuda de ferramentas de computação visual capazes de reforçar a ligação entre o estado mental e a expressão facial. Para conhecer as iniciativas existentes, catalogá-las e identificá-las, foram efetuadas buscas nas bases PubMed, Web of Science e Google Scholar, utilizando os termos: autismo, computação, imagens, expressões faciais e reconhecimento de emoções. Nos resultados obtidos pelas buscas, foram selecionados somente trabalhos que descrevem ferramentas e softwares voltados à minimização do déficit apresentado por autistas no reconhecimento e produção de expressões faciais, com ano de publicação entre 2000 e 2015 . No total, foram encontrados vinte e um trabalhos que atenderam aos critérios definidos. 
Para obter uma visão geral das publicações encontradas, a Tabela 1 apresenta os artigos ordenados por data de publicação, avaliados a partir dos seguintes critérios: público alvo; forma de aprendizado de expressões faciais; tecnologia e interatividade; expressões faciais utilizadas e funcionalidades para avaliação da evolução do paciente. Em seguida, cada um dos critérios avaliados é detalhado (Seções 2.1 a 2.5).

\subsection{Público Alvo}

A escolha do público-alvo correto é um dos fatores chave para o sucesso de ferramentas computacionais para treinamento no reconhecimento e produção de expressões faciais. Como o público autista varia sob diversos aspectos - de acordo com a gravidade do quadro e idade dos indivíduos, por exemplo - acredita-se que funcionalidades e interfaces desenvolvidas de acordo com as preferências específicas de uma população possam gerar melhores resultados.

Ao avaliar o público alvo, o primeiro critério utilizado para comparação é a faixa etária. Como o autismo não tem cura e está presente durante toda a vida do indivíduo, é importante que ferramentas sejam definidas com contexto apropriados para cada faixa etária. A importância da definição da idade apropriada está relacionada ao fato de que crianças autistas possuem interesses e capacidades motoras diferentes de jovens e adultos autistas.

Outro critério considerado para comparação é a gravidade do transtorno. Neste quesito, baseado no DSM-5 [American Psychiatric Association 2013], o autismo pode ser dividido em três níveis: exige apoio (1), exige apoio substancial (2) e exige apoio muito substancial (3). A principal diferença entre estes níveis é o grau de comprometimento das funções básicas. Autistas do nível 1 apresentam nível intelectual dentro da normalidade e déficit intelectual leve ou inexistente, possibilitando sua alfabetização. Os autistas do nível 2 e 3 são indivíduos com os casos mais grave do transtorno, onde o déficit nas características afetadas pelo transtorno é maior, e geralmente está associado a um grau maior de deficiência intelectual, o que acaba comprometendo suas habilidades de comunicação e aprendizado.

Conforme apresentado no comparativo da Tabela 1, sete dos vinte e um trabalhos são focados em autistas do nível 1. Têm-se ainda os doze trabalhos que focam no público autista geral, porém apresentam interfaces onde a alfabetização do indivíduo é necessária para sua utilização, excluindo autistas do nível 2 e 3 do público de usuários. Existem ainda duas ferramentas que podem ser utilizadas por autistas do nível 2 e 3, porém estes trabalhos representam uma minoria, haja vista as dificuldades apresentadas no desenvolvimento de ferramentas computacionais para indivíduos que apresentam limitações profundas em suas habilidades cognitivas, de comunicação e de aprendizado.

Avaliando a faixa etária do público alvo, percebe-se que dezoito dos vinte e um trabalhos têm foco em crianças, enquanto três deles estudam adolescentes e jovens adultos autistas. Acredita-se que as ferramentas sejam focadas em crianças devido à crescente demanda por instrumentos de reabilitação para o público autista infantil, o que foi impulsionado pelo incremento diagnósticos precoces nos últimos anos [Matson and Kozlowski 2011]. Outro ponto importante é que a infância é a fase mais adequada para intervenções focadas no treinamento de expressões faciais e emoções, apresentando melhores resultados quando comparadas com intervenções efetuadas em 
jovens e adultos [Aman 2005].

\subsection{Forma de treinamento de expressões faciais}

A forma de treinamento de expressões faciais compreende a metodologia utilizada para desenvolvimento de exercícios que melhorem a assertividade nas habilidades de autistas em reconhecer e produzir expressões faciais. Estas metodologias baseiam-se em achados da literatura médica, onde através de experimentos são demonstradas a efetividade dos métodos para treinamento de expressões faciais.

Categorizando-se as metodologias, pode-se estabelecer duas formas principais para treinar expressões faciais: reconhecimento de expressões faciais e produção de expressões faciais. No reconhecimento, são apresentados conteúdos (texto, imagens, sons ou vídeos) que estão associados a uma determinada emoção. A natureza dos conteúdos exibidos varia desde imagens da face de seres humanos até diálogos de situações sociais. Este conteúdo apresentado deve ser relacionado com uma expressão facial pelo autista. Esta metodologia é utilizada tanto em intervenções utilizando computadores quanto em intervenções através de fotografias, haja vista sua facilidade para ser executada.

O treinamento através da produção das expressões faciais é um tópico que vem sendo explorados em estudos recentes [Deriso et al. 2012, Gordon et al. 2014]. Esta metodologia só foi passível de inclusão em ferramentas computacionais devido aos avanços em métodos de visão computacional, que permitiram que computadores reconheçam expressões faciais em tempo real. A diferença nela é que ao invés do indivíduo reconhecer a expressão facial a partir de uma imagem, ele deve produzir, em sua própria face, a expressão requerida. O treinamento através de produção foi comprovado como sendo eficaz na melhoria das habilidades de reconhecimento de expressões faciais em [Deriso et al. 2012] e [Gordon et al. 2014]. Ainda, [Oberman et al. 2007] comprova que nossa habilidade de reconhecer expressões faciais está diretamente ligada com a habilidade de imitação das mesmas, o que reforça a importância da produção de expressões faciais.

Com base na Tabela 1, é possível visualizar que dezesseis das vinte e uma ferramentas encontradas treinam expressões faciais através do reconhecimento, enquanto somente cinco dos vinte e um trabalhos promovem o treinamento através da produção de expressões faciais. Acredita-se que esses resultados estejam relacionados com os esforços necessários para implementação do treinamento através da produção de expressões faciais, que só é possível ao implementar-se métodos de visão computacional para certificar-se que a expressão produzida está correta.

\subsection{Tecnologia e interatividade}

A tecnologia utilizada e sua forma de interação merece atenção ao desenvolver softwares para o público autista. Autistas apresentam atraso no desenvolvimento de habilidades motoras, com consequente dificuldade para a manipulação de objetos presentes na interação com computadores tradicionais, como mouses e teclados [Jasmin et al. 2009]. Esses problemas motores motivam o desenvolvimento de estratégias alternativas de interação, como telas sensíveis ao toque e métodos não motores utilizando câmeras.

Com base na Tabela 1, observa-se que quatorze dos vinte e um trabalhos foram desenvolvidos na plataforma Desktop, porém este padrão está sendo mudado ao longo 
dos anos. Devido à popularização de novos dispositivos, como tablets e óculos de realidade virtual, novas ferramentas vêm se tornando disponíveis para auxiliar autistas no treinamento de expressões faciais [Xu and Tanaka 2014]. Visualiza-se uma mudança semelhante do ponto de vista da interação com o usuário, sendo que cada vez mais interfaces multimodais são utilizadas ao invés dos tradicionais mouses e teclados. Dez das vinte e uma ferramentas já utilizam interfaces multimodais para comunicação com o usuário, representando quarenta e sete por cento dos trabalhos. Essas interfaces estão

\begin{tabular}{|c|c|c|c|c|c|c|c|c|c|}
\hline Artigo & $\mathrm{N}$ & I & FA & FI & $\mathrm{P}$ & $\mathrm{EF}$ & $\mathrm{CS}$ & RAP & CDP \\
\hline [Silver and Oakes 2001] & G & $\mathrm{C}, \mathrm{O}$ & $\mathrm{R}$ & MT & $\mathrm{D}$ & FTRS & $\mathrm{X}$ & & \\
\hline [Bölte et al. 2002] & 1 & $\mathrm{O}, \mathrm{U}$ & $\mathrm{R}$ & MT & $\mathrm{D}$ & FTRNMSE & $\mathrm{X}$ & & \\
\hline [Tanaka et al. 2003] & G & $\mathrm{C}$ & $\mathrm{R}$ & MT & $\mathrm{D}$ & FTRNM & & & $\mathrm{X}$ \\
\hline [Moore et al. 2005] & G & $\mathrm{C}, \mathrm{O}$ & $\mathrm{R}$ & MT & $\mathrm{D}$ & FTRM & $\mathrm{X}$ & & \\
\hline [Beaumont and Sofronoff 2008] & 1 & $\mathrm{C}$ & $\mathrm{R}$ & MT & $\mathrm{D}$ & FTRNMS & $\mathrm{X}$ & & \\
\hline [Cockburn et al. 2008] & $\mathrm{G}$ & $\mathrm{C}$ & $\mathrm{P}$ & $\mathrm{C}$ & $\mathrm{D}$ & $\mathrm{F}$ & & & \\
\hline [Grynszpan et al. 2008] & 1 & $\mathrm{C}$ & $\mathrm{R}$ & MT & $\mathrm{D}$ & HSAFSD & $\mathrm{X}$ & & \\
\hline [Madsen et al. 2008] & 1 & $\mathrm{C}$ & $\mathrm{R}$ & $\mathrm{C}, \mathrm{TS}$ & $\mathrm{M}$ & Complex. & & & \\
\hline [Finkelstein et al. 2009] & G & $\mathrm{C}, \mathrm{O}$ & $\mathrm{R}$ & MT & $\mathrm{D}$ & FTRNMS & $\mathrm{X}$ & & \\
\hline [Abirached et al. 2011] & $\mathrm{G}$ & $\mathrm{C}, \mathrm{O}$ & $\mathrm{R}, \mathrm{P}$ & $\mathrm{C}, \mathrm{TS}$ & $\bar{M}$ & FTRNMS & $\mathrm{X}$ & & \\
\hline [Hopkins et al. 2011] & 1,2 & $\mathrm{C}$ & $\mathrm{R}$ & MT & $\mathrm{D}$ & FTRNMS & & & \\
\hline [Deriso et al. 2012] & $\mathrm{G}$ & $\mathrm{C}$ & $\mathrm{P}$ & $\mathrm{C}$ & $\mathrm{D}$ & FTRNMS & $\mathrm{X}$ & & \\
\hline [Ryokai et al. 2012] & G & $\mathrm{C}$ & $\mathrm{P}$ & $\mathrm{C}, \mathrm{TS}$ & M & - & $\mathrm{X}$ & & \\
\hline [Bekele et al. 2013] & $\mathrm{G}$ & $\mathrm{O}$ & $\mathrm{R}$ & MT & $\mathrm{D}$ & FTRNMSD & & & $\mathrm{X}$ \\
\hline [Gay et al. 2013] & $\bar{G}$ & $\mathrm{C}$ & $\mathrm{R}$ & $\mathrm{C}, \mathrm{TS}$ & $\mathrm{M}$ & FTRNMS & $\mathrm{X}$ & $\mathrm{X}$ & \\
\hline [Kandalaft et al. 2013] & 1 & $\mathrm{U}$ & $\mathrm{R}$ & MT & $\mathrm{D}$ & - & $\mathrm{X}$ & & \\
\hline [Christinaki et al. 2014] & G & $\mathrm{C}$ & $\mathrm{R}$ & $\mathrm{C}$ & $\mathrm{C}$ & FTRMS & & & \\
\hline [Harrold et al. 2014] & 1 & $\mathrm{C}$ & $\mathrm{P}$ & $\mathrm{C}$ & M & FTRNMS & & & \\
\hline [Serret et al. 2014] & 1,2 & $\mathrm{C}$ & $\mathrm{R}$ & MT & $\mathrm{C}$ & FTRNMSOE & & & \\
\hline [Wang et al. 2014] & $\mathrm{G}$ & $\mathrm{C}$ & $\mathrm{R}$ & ORV & $\mathrm{D}$ & - & & & \\
\hline [Chen et al. 2015] & 1 & $\mathrm{C}$ & $\mathrm{R}$ & $\mathrm{C}$ & $\mathrm{D}$ & FTRNMS & $\mathrm{X}$ & & \\
\hline
\end{tabular}

Table 1. Comparação entre os trabalhos para treinamento de expressões faciais.

- Cabeçalho: N - Nível; I - Idade; FA - Forma de Aprendizado; FI - Forma de Interação; P - Plataforma; EF - Expressões Faciais; CS - Contexto Social; RAP - Relatório para Acompanhamento do Paciente; CDP - Coleta de Dados do Paciente.

- Nível: G - Geral; 1- Exige apoio; 2- Exige apoio substancial; 3- Exige apoio muito substancial.

- Idade: C - Crianças; O - Adolescentes; U - Adultos.

- Forma de Aprendizado: R - Reconhecimento; P - Produção.

- Forma de Interação: C - Câmera; ORV - Óculos para Realidade Virtual; TS - Touch Screen; MT - Mouse e Teclado.

- Plataforma: D - Desktop; ORV - Óculos para Realidade Virtual; C - Console; M Dispositivos Móveis.

- Exp. Faciais: F - felicidade; T - tristeza; R - raiva, N - nojo, M - medo; S - surpresa; D desprezo; O - dor; E - Neutro; Complex - Emoções complexas. 
sendo utilizadas com auxilio da visão computacional, através do reconhecimento de gestos e expressões nos utilizadores com ajuda de câmeras de vídeo.

\subsection{Expressões faciais}

Ao treinar habilidades de reconhecimento e produção de expressões faciais em autistas, é importante definir quais expressões faciais serão utilizadas. Conforme mencionado previamente, autistas apresentam limitações na formação da teoria da mente, decorrentes de dificuldades no reconhecimento e produção de expressões faciais. Tais expressões englobam não apenas as seis básicas definidas por Ekman (felicidade, tristeza, surpresa, medo, raiva e nojo) [Ekman and Friesen 1978], mas também expressões complexas que podem representar dor [Minio-Paluello et al. 2009].

Com a mesma importância que o reconhecimento da expressão facial para interpretação de sua emoção associada, está o entendimento do contexto social em que ela está inserida. Por exemplo, uma pessoa em uma situação amedrontadora demonstrando raiva pode ser compreendida como expressando medo por observadores [Carroll and Russell 1996]. Autistas também apresentam limitações no entendimento de situações sociais em comparação com indivíduos não autistas [Santos et al. 2012], o que dificulta o entendimento de pistas importantes para reconhecimento da expressão facial e de seu significado. Quanto a estas pistas, seu treinamento utilizando ferramentas computacionais é efetivo em autistas, visto que podem prover um ambiente controlado para que ele pratique suas habilidades sem a pressão e imprevisibilidade do mundo real.

Dos vinte e um trabalhos encontrados, três não apresentaram dados sobre quais as expressões faciais utilizadas. Dezessete dos vinte e um trabalhos utilizaram as seis expressões faciais básicas descritas por Ekman [Ekman and Friesen 1978], ou uma variação das mesmas. Entre as variações encontradas, podem-se citar seis trabalhos que removeram uma ou mais expressões faciais. Outra variação das seis expressões faciais básicas é a inclusão de expressões como as associadas a dor [Serret et al. 2014] e desprezo [Bekele et al. 2013]. Como variação total das expressões básicas definidas por Ekman, pode-se citar o trabalho de [Madsen et al. 2008], que ensina seis expressões faciais complexas.

\subsection{Funcionalidades para avaliação da evolução do paciente}

Além das funcionalidades para o treinamento de expressões faciais, ferramentas computacionais podem prover funcionalidades importantes para que profissionais da área de saúde possam acompanhar a evolução de seus pacientes. Estas funcionalidades também possibilitam o registro longitudinal de dados que podem contribuir para um melhor entendimento do problema. As informações utilizadas para o acompanhamento podem ser de diversos tipos, como dados sobre o tempo para execução de determinado desafio, informações sobre erros e acertos do paciente ao executar determinada tarefa e vídeos do paciente utilizando a ferramenta. Para acessar estas informações de forma simplificada, relatórios são incluídos nas ferramentas, possibilitando seu acesso pelo médico ou terapeuta responsável. Estes relatórios devem estar devidamente protegidos por mecanismos de autenticação, garantindo a privacidade do paciente.

Para obter mais informações sobre a presença de relatórios para visualização de dados obtidos nas ferramentas, foram buscadas menções que descrevam esta 
funcionalidade nos trabalhos encontrados. Conforme apresentado na Tabela 1, somente a pesquisa de [Gay et al. 2013] conta com um relatório para acompanhamento da evolução do paciente. Esse relatório apresenta informações sobre os acertos e erros do paciente ao reconhecer as expressões faciais.

No contexto da coleta de dados dos pacientes, os únicos trabalhos que apresentam alguma funcionalidade são os desenvolvidos por Bekele [Bekele et al. 2013] e Tanaka [Tanaka et al. 2003]. Em [Bekele et al. 2013], são utilizados equipamentos para coletar dados fisiológicos e de rastreamento ocular do paciente ao reconhecer a expressão facial apresentada. Já no trabalho de Tanaka [Tanaka et al. 2003], foi desenvolvido um mecanismo de coleta de dados do paciente em um estudo mais moderno da ferramenta [Tanaka et al. 2010], porém os dados coletados são somente sobre erros e acertos do paciente.

\section{Discussão}

Com base nas ferramentas encontradas e avaliadas com o objetivo de treinar habilidades de reconhecimento e produção de expressões faciais por autistas, é possível concluir que este campo está sendo aperfeiçoado ao longo dos anos, de diferentes formas. Observando o comparativo entre os trabalhos, percebe-se que essas melhorias foram efetuadas principalmente nos critérios de plataforma e interatividade, tornando as ferramentas mais inclusivas e facilitando o seu uso pelo público autista. Apesar de haver melhorias significativas em alguns critérios, percebe-se pouca atenção para a evolução de outros quesitos.

Do ponto de vista tecnológico, é possível visualizar que nenhuma das ferramentas foi desenvolvida para a plataforma web. Esta plataforma apresenta distribuição e acesso simplificados, não necessitando da instalação de nenhum software além do navegador de internet para sua utilização. Como navegadores estão disponíveis na maioria dos dispositivos, ferramentas desenvolvidas na plataforma web podem ser utilizadas em notebooks, celulares e tablets. Com o recente avanço do padrão HTML5, o acesso a câmeras e o armazenamento de dados no navegador de internet foram simplificados, permitindo que ferramentas computacionais explorem estes recursos.

Ao avaliar a metodologia para treinamento de expressões faciais, percebe-se que a produção de expressões faciais vem ganhando espaço, sendo explorada como alternativa aos métodos tradicionais de reconhecimento de expressões faciais. Tanto o reconhecimento [Tanaka et al. 2010] quanto a produção [Deriso et al. 2012] possuem trabalhos na literatura que comprovam sua efetividade na melhoria das habilidades de reconhecimento de expressões faciais. Acredita-se que a combinação de ambos seja o melhor caminho, provendo diferentes exercícios para o treinamento no reconhecimento e produção de expressões faciais.

Do ponto de vista da psicologia, a grande maioria das ferramentas continuam utilizando somente as seis expressões faciais básicas definidas por Ekman. No campo da psicologia, o conhecimento sobre expressões faciais vem sendo aperfeiçoando através de novas descobertas. Atualmente, existem trabalhos apontando que expressões faciais e seu entendimento não são culturalmente universais [Gendron et al. 2014], o que reforça a ideia de que ferramentas devem ser customizadas para seu público alvo. Fatores como contexto social em que a expressão facial é apresentada devem ser levados em conta no 
desenvolvimento dos desafios das ferramentas.

No quesito de dados para avaliação da evolução do paciente, nota-se pouca atenção para o desenvolvimento dessas funcionalidades nas ferramentas encontradas. Somente o trabalho de [Gay et al. 2013] apresentou relatórios para visualização deste tipo de dados. Estes relatórios são extremamente importantes para profissionais da área da saúde, porque permitem que o profissional tenha acesso, de forma simples, aos dados coletados sobre a utilização da ferramenta pelo paciente. Deve-se ressaltar a importância da inclusão de relatórios que permitam que esses dados sejam avaliados de forma global, auxiliando no entendimento de questões relacionadas ao transtorno autista como um todo.

Aprofundando-se nos dados coletados para avaliação da evolução do paciente, outros dados não relacionados a acertos e erros nas tarefas podem ser processados por ferramentas computacionais. Como exemplo, pode-se citar a gravação de vídeos do usuário executando desafios e informações sobre a trajetória do rastreamento ocular ao reconhecer uma expressão facial. Quanto aos vídeos gravados, seu principal uso seria na futura avaliação do paciente e da população autista em geral. Como os vídeos de utilização das ferramentas podem ser muito longos, tornando a análise laboriosa, acreditase no potencial de métodos de computação visual para sumarizar e extrair as partes mais relevantes dos vídeos.

Quanto ao rastreamento ocular, diversos trabalhos na literatura reforçam sua importância como critério para melhor entendimento do autismo [Falck-Ytter et al. 2013]. Focando na temática de expressões faciais, sabese que o rastreamento ocular é eficaz para auxiliar na compreensão do método utilizado por pacientes autistas para reconhecer expressões faciais [Bekele et al. 2014, Grynszpan et al. 2012]. Em ferramentas computacionais, seu uso pode ser ainda maior, visto que o percurso efetuado pelo autista durante a realização dos desafios propostos pode ser utilizado para otimizar a interface da ferramenta.

A implementação do rastreamento ocular é um desafio enfrentando pela área de visão computacional, sendo que [Hansen and Ji 2010] apresentam uma revisão de literatura dos principais métodos utilizados para resolução do problema. Existem hardwares específicos para efetuar rastreamento ocular disponíveis no mercado, como o Tobii X120 utilizado em [Bekele et al. 2013], que apresentam alta acurácia e confiabilidade nos dados obtidos. Apesar dos pontos positivos, este tipo de equipamento apresenta alto custo e não está integrado na maioria dos computadores. Webcams e câmeras de profundidade também podem ser utilizadas como dispositivos para rastreamento ocular, obtendo acurácia e confiabilidade similares aos dispositivos comerciais [San Agustin et al. 2010].

Baseado na melhoria dos aspectos apontados, visualiza-se um caminho futuro de novas ferramentas para treinamento de em reconhecimento e produção de expressões faciais por autistas. Ao incorporar as melhorias nos critérios citados, as novas ferramentas desenvolvidas possuem potencial na modernizando de intervenções terapêuticas para incremento das habilidades de pacientes autistas em reconhecer e produzir expressões faciais. 


\section{Conclusão}

Este trabalho apresenta uma revisão dos estudos que utilizam recursos computacionais no desenvolvimento de ferramentas para o treinamento no reconhecimento e produção de expressões faciais por autistas, procurando oferecer uma visão crítica da área para novos pesquisadores. Foram encontrados vinte e um artigos que satisfizeram as condições buscadas, sendo eles avaliados com base em cinco critérios: público alvo; forma de aprendizado de expressões faciais; forma de interação com a ferramenta; expressões faciais utilizadas e funcionalidades para avaliação da evolução do paciente.

Com base no trabalho comparativo efetuado, este artigo apresenta critérios de melhoria em quatro dos cinco quesitos avaliados nas ferramentas. Entre as melhorias, são citadas as seguintes: inclusão de novos conceitos descobertos pela área de psicologia; inclusão da produção de expressões faciais como parte do treinamento; utilização da tecnologia web para permitir a portabilidade e o acesso simplificado às ferramentas; e a inclusão de funcionalidades médicas para obtenção de dados que auxiliem na avaliação da evolução do paciente, como a captura de vídeos do usuário e dados de rastreamento ocular ao avaliar determinada expressão facial.

Como um dos trabalhos futuros na área, aponta-se para o desenvolvimento de uma ferramenta computacional em um projeto multidisciplinar, envolvendo profissionais das áreas de ciência da computação e ciências da saúde. Esta ferramenta deve incorporar as melhorias nos critérios apontados, possibilitando uma intervenção moderna e eficiente. Através da ferramenta, o profissional responsável poderá acompanhar o progresso do paciente ao longo de sua utilização, possibilitando avaliar sua evolução e adequar a intervenção para as necessidades daquele paciente.

\section{References}

Abirached, B., Zhang, Y., Aggarwal, J. K., Tamersoy, B., Fernandes, T., Miranda, J., and Orvalho, V. (2011). Improving communication skills of children with asds through interaction with virtual characters. In Serious Games and Applications for Health (SeGAH), 2011 IEEE 1st International Conference on, pages 1-4. IEEE.

Aman, M. G. (2005). Treatment planning for patients with autism spectrum disorders. Journal of Clinical Psychiatry, 66:38.

American Psychiatric Association (2013). Diagnostic and statistical manual of mental disorders: DSM-5. Autor, Washington, DC, 5th ed. edition.

Baron-Cohen, S., Leslie, A. M., and Frith, U. (1985). Does the autistic child have a "theory of mind?". Cognition, 21(1):37-46.

Beaumont, R. and Sofronoff, K. (2008). A multi-component social skills intervention for children with asperger syndrome: The junior detective training program. Journal of Child Psychology and Psychiatry, 49(7):743-753.

Bekele, E., Crittendon, J., Zheng, Z., Swanson, A., Weitlauf, A., Warren, Z., and Sarkar, N. (2014). Assessing the utility of a virtual environment for enhancing facial affect recognition in adolescents with autism. Journal of autism and developmental disorders, 44(7):1641-1650. 
Bekele, E., Zheng, Z., Swanson, A., Crittendon, J., Warren, Z., and Sarkar, N. (2013). Understanding how adolescents with autism respond to facial expressions in virtual reality environments. Visualization and Computer Graphics, IEEE Transactions on, 19(4):711-720.

Bölte, S., Feineis-Matthews, S., Leber, S., Dierks, T., Hubl, D., and Poustka, F. (2002). The development and evaluation of a computer-based program to test and to teach the recognition of facial affect. International Journal of Circumpolar Health, 61.

Carroll, J. M. and Russell, J. A. (1996). Do facial expressions signal specific emotions? judging emotion from the face in context. Journal of personality and social psychology, $70(2): 205$.

Chen, C.-H., Lee, I.-J., and Lin, L.-Y. (2015). Augmented reality-based self-facial modeling to promote the emotional expression and social skills of adolescents with autism spectrum disorders. Research in developmental disabilities, 36:396-403.

Christinaki, E., Vidakis, N., and Triantafyllidis, G. (2014). A novel educational game for teaching emotion identification skills to preschoolers with autism diagnosis. Computer science and information systems, 11(2):723-743.

Cockburn, J., Bartlett, M., Tanaka, J., Movellan, J., Pierce, M., and Schultz, R. (2008). Smilemaze: A tutoring system in real-time facial expression perception and production in children with autism spectrum disorder. In ECAG 2008 Workshop Facial and Bodily Expressions for Control and Adaptation of Games, page 3.

Deriso, D. M., Susskind, J., Tanaka, J., Winkielman, P., Herrington, J., Schultz, R., and Bartlett, M. (2012). Exploring the facial expression perception-production link using real-time automated facial expression recognition. In Computer Vision-ECCV 2012. Workshops and Demonstrations, pages 270-279. Springer.

Ekman, P. and Friesen, W. V. (1978). Manual for the facial action coding system. Consulting Psychologists Press.

Falck-Ytter, T., Bölte, S., and Gredebäck, G. (2013). Eye tracking in early autism research. Journal of neurodevelopmental disorders, 5(1):28.

Finkelstein, S. L., Nickel, A., Harrison, L., Suma, E., Barnes, T., et al. (2009). cmotion: A new game design to teach emotion recognition and programming logic to children using virtual humans. In Virtual Reality Conference, 2009. VR 2009. IEEE, pages 249-250. IEEE.

Gay, V., Leijdekkers, P., Agcanas, J., Wong, F., and Wu, Q. (2013). Capturemyemotion: helping autistic children understand their emotions using facial expression recognition and mobile technologies. BLED 2013 Proceedings.

Gendron, M., Roberson, D., van der Vyver, J. M., and Barrett, L. F. (2014). Perceptions of emotion from facial expressions are not culturally universal: evidence from a remote culture. Emotion, 14(2):251.

Gordon, I., Pierce, M. D., Bartlett, M. S., and Tanaka, J. W. (2014). Training facial expression production in children on the autism spectrum. Journal of autism and developmental disorders, 44(10):2486-2498. 
Grynszpan, O., Martin, J.-C., and Nadel, J. (2008). Multimedia interfaces for users with high functioning autism: An empirical investigation. International Journal of HumanComputer Studies, 66(8):628-639.

Grynszpan, O., Nadel, J., Martin, J.-C., Simonin, J., Bailleul, P., Wang, Y., Gepner, D., Le Barillier, F., and Constant, J. (2012). Self-monitoring of gaze in high functioning autism. Journal of autism and developmental disorders, 42(8):1642-1650.

Hansen, D. W. and Ji, Q. (2010). In the eye of the beholder: A survey of models for eyes and gaze. Pattern Analysis and Machine Intelligence, IEEE Transactions on, 32(3):478-500.

Harrold, N., Tan, C. T., Rosser, D., and Leong, T. W. (2014). Copyme: a portable real-time feedback expression recognition game for children. In CHI'14 Extended Abstracts on Human Factors in Computing Systems, pages 1195-1200. ACM.

Hopkins, I. M., Gower, M. W., Perez, T. A., Smith, D. S., Amthor, F. R., Wimsatt, F. C., and Biasini, F. J. (2011). Avatar assistant: improving social skills in students with an asd through a computer-based intervention. Journal of autism and developmental disorders, 41(11):1543-1555.

Jasmin, E., Couture, M., McKinley, P., Reid, G., Fombonne, E., and Gisel, E. (2009). Sensori-motor and daily living skills of preschool children with autism spectrum disorders. Journal of autism and developmental disorders, 39(2):231-241.

Kandalaft, M. R., Didehbani, N., Krawczyk, D. C., Allen, T. T., and Chapman, S. B. (2013). Virtual reality social cognition training for young adults with high-functioning autism. Journal of Autism and Developmental Disorders, 43(1):34-44.

Klin, A. (2006). Autismo e síndrome de asperger: uma visão geral. Revista Brasileira de Psiquiatria, 28(1):3-11.

Madsen, M., El Kaliouby, R., Goodwin, M., and Picard, R. (2008). Technology for justin-time in-situ learning of facial affect for persons diagnosed with an autism spectrum disorder. In Proceedings of the 10th international ACM SIGACCESS conference on Computers and accessibility, pages 19-26. ACM.

Matson, J. L. and Kozlowski, A. M. (2011). The increasing prevalence of autism spectrum disorders. Research in Autism Spectrum Disorders, 5(1):418-425.

Minio-Paluello, I., Baron-Cohen, S., Avenanti, A., Walsh, V., and Aglioti, S. M. (2009). Absence of embodied empathy during pain observation in asperger syndrome. Biological psychiatry, 65(1):55-62.

Moore, D., Cheng, Y., McGrath, P., and Powell, N. J. (2005). Collaborative virtual environment technology for people with autism. Focus on Autism and Other Developmental Disabilities, 20(4):231-243.

Oberman, L. M., Winkielman, P., and Ramachandran, V. S. (2007). Face to face: Blocking facial mimicry can selectively impair recognition of emotional expressions. Social neuroscience, 2(3-4):167-178.

Picard, R. W., Vyzas, E., and Healey, J. (2001). Toward machine emotional intelligence: Analysis of affective physiological state. Pattern Analysis and Machine Intelligence, IEEE Transactions on, 23(10):1175-1191. 
Ryokai, K., Raffle, H., and Kowalski, R. (2012). Storyfaces: pretend-play with ebooks to support social-emotional storytelling. In Proceedings of the 11th International Conference on Interaction Design and Children, pages 125-133. ACM.

San Agustin, J., Skovsgaard, H., Mollenbach, E., Barret, M., Tall, M., Hansen, D. W., and Hansen, J. P. (2010). Evaluation of a low-cost open-source gaze tracker. In Proceedings of the 2010 Symposium on Eye-Tracking Research \& Applications, pages 77-80. ACM.

Santos, A., Chaminade, T., Da Fonseca, D., Silva, C., Rosset, D., and Deruelle, C. (2012). Just another social scene: evidence for decreased attention to negative social scenes in high-functioning autism. Journal of autism and developmental disorders, 42(9):17901798.

Serret, S., Hun, S., Iakimova, G., Lozada, J., Anastassova, M., Santos, A., Vesperini, S., and Askenazy, F. (2014). Facing the challenge of teaching emotions to individuals with low-and high-functioning autism using a new serious game: a pilot study. Molecular autism, 5(1):37.

Silver, M. and Oakes, P. (2001). Evaluation of a new computer intervention to teach people with autism or asperger syndrome to recognize and predict emotions in others. Autism, 5(3):299-316.

Tanaka, J. W., Lincoln, S., and Hegg, L. (2003). A framework for the study and treatment of face processing deficits in autism. The development of face processing, pages 101119.

Tanaka, J. W., Wolf, J. M., Klaiman, C., Koenig, K., Cockburn, J., Herlihy, L., Brown, C., Stahl, S., Kaiser, M. D., and Schultz, R. T. (2010). Using computerized games to teach face recognition skills to children with autism spectrum disorder: The let's face it! program. Journal of Child Psychology and Psychiatry, 51(8):944-952.

Tanaka, J. W., Wolf, J. M., Klaiman, C., Koenig, K., Cockburn, J., Herlihy, L., Brown, C., Stahl, S. S., South, M., McPartland, J. C., et al. (2012). The perception and identification of facial emotions in individuals with autism spectrum disorders using the let's face it! emotion skills battery. Journal of Child Psychology and Psychiatry, 53(12):1259-1267.

Wang, X., Desalvo, N., Gao, Z., Zhao, X., Lerman, D. C., Gnawali, O., and Shi, W. (2014). Eye contact conditioning in autistic children using virtual reality technology. In Pervasive Computing Paradigms for Mental Health, pages 79-89. Springer.

Xu, B. and Tanaka, J. W. (2014). Teaching children with autism to recognize faces. In Comprehensive Guide to Autism, pages 1043-1059. Springer.

Zablotsky, B., Black, L., Maenner, M., Schieve, L., and Blumberg, S. (2015). Estimated prevalence of autism and other developmental disabilities following questionnaire changes in the 2014 national health interview survey. National health statistics reports, (87):1-21. 